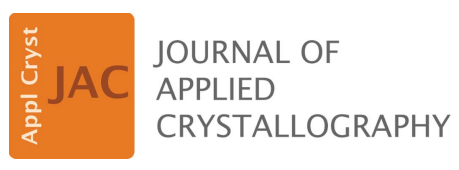

ISSN 1600-5767

Received 17 June 2021

Accepted 26 July 2021

Edited by J. M. García-Ruiz, Instituto Andaluz de Ciencias de la Tierra, Granada, Spain

Keywords: total scattering; pair distribution function.
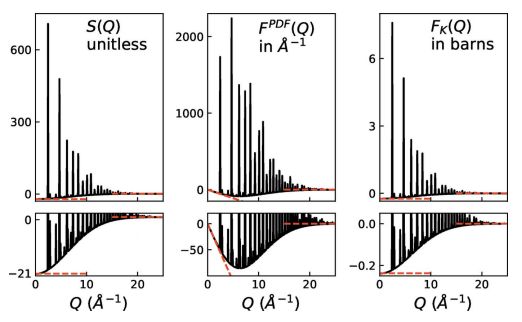

OPEN $\odot$ ACCESS

\section{Illustrated formalisms for total scattering data: a guide for new practitioners. Corrigendum and addendum}

\author{
Peter F. Peterson ${ }^{\mathrm{a}, \mathrm{b} *}$ and David A. Keen ${ }^{\mathrm{c} *}$
}

${ }^{a}$ Computer Science and Mathematics Division, Oak Ridge National Laboratory, Oak Ridge, TN, USA, ${ }^{\mathbf{b}}$ Neutron Scattering Division, Oak Ridge National Laboratory, Oak Ridge, TN, USA, and ' ${ }^{\mathrm{ISIS}}$ Facility, Rutherford Appleton Laboratory, Harwell Campus, Didcot, Oxfordshire, United Kingdom. *Correspondence e-mail: petersonpf@ornl.gov, david.keen@stfc.ac.uk

Errors and ambiguities in the article by Peterson, Olds, McDonnell \& Page [J. Appl. Cryst. (2021), 54, 317-332] are corrected and clarified, respectively.

In the article Illustrated formalisms for total scattering data: a guide for new practitioners (Peterson et al., 2021) the authors provide a detailed comparison between the various notations and formalisms used by the total scattering/pair distribution function (PDF) community. The paper repeats the relationships already established by Keen (2001), presents look-up tables for easy conversion between functions, and provides graphical examples based on calculated neutron scattering functions of liquid argon and powdered $\mathrm{MnO}$ from molecular dynamics simulations and the cubic crystal structure, respectively. However, there is a confusing choice of units when some of the functions are presented graphically, leading to a mis-labelling the $y$ axes of several of the figures. Furthermore, the low- $Q$ limits have been defined incorrectly, such that they are only applicable for monoatomic materials. Given the pedagogical nature of the article (Peterson et al., 2021), we felt it necessary to provide this corrigendum to clarify any unintended confusion. For greater clarity, we provide a worked example based on experimental GEM data from $\mathrm{BaTiO}_{3}$ (Senn et al., 2016) in support of these revisions.

As already pointed out by Keen (2001) and Peterson et al. (2021), in this field different communities lay claim to the same function names for subtly different definitions. In order to be completely explicit in this corrigendum we will use the subscript ' $K$ ' and superscript 'PDF' to clarify multiply defined functions consistent with the sub/superscripting used by Peterson et al. (2021) and Keen (2001), respectively. We have not assigned these additional labels to functions [such as $S(Q)$ and $\rho(r)]$ that are defined identically by Keen (2001) and Peterson et al. (2021). This somewhat tedious notation will be helpful when presenting clarifying points and is detailed in Table 1.

Neutron scattering lengths were typically given in units of $10^{-12} \mathrm{~cm}$, i.e. $10 \mathrm{fm}$ units, a natural working unit since neutron cross sections are expressed in barns $\left(10^{-24} \mathrm{~cm}^{2}\right)$ [for example International Tables for X-ray Crystallography, Vol. III (1962)]; the powers of ten, although implicitly present, could be ignored in practice. They are now more commonly tabulated in fm (Sears, 1992) and these are the values used by Peterson et al. (2021). This has introduced an inadvertent 
Table 1

Connection of formalisms to those found in previous work.

It is fully expected that future publications will not employ the subscript/ superscript used here, but might nonetheless refer to these specific equations when establishing notation or define equations in terms of $S(Q)$ or $\rho(r)$ which have common meaning.

\begin{tabular}{lll}
\hline Function & Peterson et al. (2021) equation & Keen (2001) equation \\
\hline$F^{\mathrm{PDF}}(Q)$ & 10 & 45 (implicit) \\
$S(Q)$ & 4 & $19 / 20$ \\
$F_{\mathrm{K}}(Q)$ & $11 / 13$ & $9 / 19$ \\
& & \\
$G^{\mathrm{PDF}}(r)$ & 19 & 43 \\
$g^{\mathrm{PDF}}(r)$ & 22 & 41 \\
$\rho(r)$ & 16 & 46 \\
$G_{\mathrm{K}}(Q)$ & $26 / 27$ & $10 / 44$ \\
\hline
\end{tabular}

scaling error in Figs. 1(e), 1(f), 2(e), 2(f), $3\left[G_{\mathrm{K}}(r)\right.$ and $\left.T(r)\right]$ and $4\left[G_{\mathrm{K}}(r)\right.$ and $\left.T(r)\right]$ of Peterson et al. (2021); the functions in the figures with $y$ axes labelled 'barn' or 'barn $/ \AA^{2}$, should be $100 \times$ smaller than presented therein. Those functions that are further scaled by $\left\langle b_{\mathrm{coh}}\right\rangle^{-2}\left[\right.$ e.g. $F^{\mathrm{PDF}}(Q), G^{\mathrm{PDF}}(r)$ or $\left.\rho(r)\right]$ are unaffected as the scattering length units cancel out.

The low- $Q$ limit of total scattering structure factors is related in a complex way to thermodynamic functions and fluctuations [see for example Table 30.2 of Cusack (1987)] most of which, for 'well behaved' non-magnetic systems, are assumed to give a small correction to the expected level. It can be further complicated by other sources of low- $Q$ 'small-angle' scattering, such as from longer-ranged scattering density variations (e.g. from nanoparticles) and magnetism, all of which are ignored in the following derivations. The low- $Q$ limit of the partial total scattering structure factors, $A_{i j}(Q)$, is
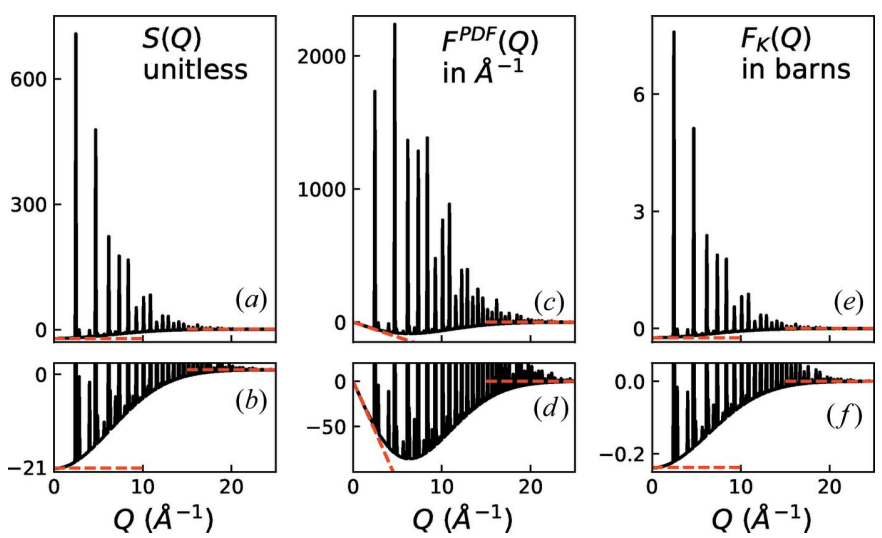

Figure 1

Redrawn version of Fig. 1 of Peterson et al. (2021) with corrected limiting values and scaling. The figure shows the comparison between various reciprocal-space neutron total scattering data from MnO: $(a),(b) S(Q)$; $(c),(d) F^{\mathrm{PDF}}(Q)$; and $(e),(f) F_{\mathrm{K}}(Q)$. The upper plots show an overview of the various functions, although the height of the Bragg peaks is mostly dictated by the width of the resolution function used (the same width was used in all plots). The asymptotes at high and low $Q$ are highlighted with red dashed lines and pertinent values are given in Table 4 . The low- $Q$ limit of $F^{\mathrm{PDF}}(Q)$ is a sloped line which only remains linear for an extended range of $Q$ because these are synthetic data with infinite sample size (and no magnetic contribution). However, the same behaviour holds true for highly ordered $\mathrm{BaTiO}_{3}$ in Fig. 2. Note that contributions from the terms involving $\eta$ for $\mathrm{MnO}$ are effectively zero on the scale of these figures.
Table 2

Limits of reciprocal-space functions.

This is a corrected version of Table 3 of Peterson et al. (2021) with all low- $Q$ limits changed and monatomic low- $Q$ limits added. The 'low- $Q$ limit' column is correct for all materials (noting that these expressions are only approximately followed for measurements of real materials and assume there is no additional 'small-angle' scattering) and the 'monatomic low- $Q$ limit' largely replicates the values in the original article. For solids and many liquids, $\eta$ is usually considered sufficiently close to zero to be ignored, especially given that these low- $Q$ limits should only be treated as indicative for real materials.

\begin{tabular}{lllll}
\hline Function & Low $Q$ & Monatomic low $Q$ & High $Q$ & Units \\
\hline$S(Q)$ & $\eta+1-\left\langle b_{\text {coh }}^{2}\right\rangle /\left\langle b_{\text {coh }}\right\rangle^{2}$ & $\eta$ & 1 & Unitless \\
$F^{\mathrm{PDF}}(Q)$ & 0 & 0 & 0 & $\AA^{-1}$ \\
$F_{\mathrm{K}}(Q)$ & $\left\langle b_{\mathrm{coh}}\right\rangle^{2} \eta-\left\langle b_{\mathrm{coh}}^{2}\right\rangle$ & $b_{\mathrm{coh}}^{2}(\eta-1)$ & 0 & Barn \\
\hline
\end{tabular}

given in equation (13) of Keen (2001) and by McGreevy \& Mitchell (1982) and is frequently shown in experimental data (Fischer et al., 2006; Bowron et al., 2006):

$$
\lim _{Q \rightarrow 0} A_{i j}(Q)-1 \simeq \rho_{0} k_{\mathrm{B}} T \kappa_{\mathrm{T}}-\delta_{i j} / c_{i},
$$

where the symbols have their usual meanings [e.g. in the text following equation (8) of Peterson et al. (2021)]. The first term on the right-hand side of equation (1) is the isothermal compressibility term, $\eta$, of Bhatia \& Thornton (1970). It is usually small relative to the other terms in the low- $Q$ limit of the total scattering structure factors (given below) and frequently treated as zero for data normalization purposes (although note that this may not always be the case and it may provide useful physical insight; Cusack, 1987). $A_{i j}(Q)$ is defined identically by Keen (2001) and Peterson et al. (2021). Since

$$
F_{\mathrm{K}}(Q)=\sum_{i, j=1}^{n} c_{i} c_{j} \bar{b}_{i} \bar{b}_{j}\left[A_{i j}(Q)-1\right],
$$

this gives rise to the following low- $Q$ limits for the total structure factors,

$$
\lim _{Q \rightarrow 0} F_{\mathrm{K}}(Q) \simeq\left(\sum_{i=1}^{n} c_{i} \bar{b}_{i}\right)^{2} \eta-\sum_{i=1}^{n} c_{i} \bar{b}_{i}^{2}=\left\langle b_{\mathrm{coh}}\right\rangle^{2} \eta-\left\langle b_{\mathrm{coh}}^{2}\right\rangle,
$$

and with $S(Q)=F_{\mathrm{K}}(Q) /\left\langle b_{\mathrm{coh}}\right\rangle^{2}+1$ yields

$$
\lim _{Q \rightarrow 0} S(Q) \simeq \eta-\frac{\left\langle b_{\text {coh }}^{2}\right\rangle}{\left\langle b_{\text {coh }}\right\rangle^{2}}+1 .
$$

Here we have explicitly propagated $\eta$ through the equations above, rather than using it more flexibly [as was done by Keen (2001)], whilst still bearing in mind that the definition in equation (1) is not valid in all circumstances (Cusack, 1987). These limits are different from those given in equations (7) and (64) and Table 3 of Peterson et al. (2021), which are only valid for monatomic systems. The corrected behaviour for various total scattering structure factors is summarized in Table 2, and the limits are recalculated using the average neutron scattering length constants in Table 3 to give the results in Table 4. Taking these points together, and as an example, we show in Fig. 1 a corrected version of Fig. 1 of Peterson et al. (2021) with very different low- $Q$ limiting values and a much-reduced $y$-axis scale for $F_{\mathrm{K}}(Q)$. Fig. 2 and the plots 
Table 3

Average neutron scattering length constants for selected materials calculated using neutron scattering lengths and cross sections provided by Sears (1992).

Note that 1 barn $=10^{-24} \mathrm{~cm}^{2}=100 \mathrm{fm}^{2}$.

\begin{tabular}{lllll}
\hline $\begin{array}{l}\text { Peterson } \text { et al. } \\
(2021) \text { notation }\end{array}$ & $\begin{array}{l}\text { Keen }(2001) \\
\text { notation }\end{array}$ & $\begin{array}{l}\mathrm{MnO} \text { value } \\
\text { (barn) }\end{array}$ & $\begin{array}{l}\mathrm{BaTiO}_{3} \text { value } \\
\text { (barn) }\end{array}$ & $\begin{array}{l}\mathrm{SiO}_{2} \text { value } \\
\text { (barn) }\end{array}$ \\
\hline$\left\langle b_{\text {tot }}^{2}\right\rangle$ & $\sum_{i=1}^{n} c_{i} \overline{b_{i}^{2}}$ & 0.254 & 0.325 & 0.282 \\
$\left\langle b_{\text {coh }}^{2}\right\rangle$ & $\sum_{i=1}^{n} c_{i} \bar{b}_{i}^{2}$ & 0.238 & 0.277 & 0.282 \\
$\left\langle b_{\text {coh }}\right\rangle^{2}$ & $\left(\sum_{i=1}^{n} c_{i} \bar{b}_{i}\right)^{2}$ & 0.011 & 0.145 & 0.276 \\
\hline
\end{tabular}

of $G_{\mathrm{K}}(r)$ and $T(r)$ in Figs. 3 and 4 in the original article need to be similarly modified but are not included here.

The above discussion also highlights another important point. Although the scaled functions $\left[S(Q), F^{\mathrm{PDF}}(Q)\right.$ and $G^{\mathrm{PDF}}(r)$ etc., which are divided through by $\left.\left\langle b_{\mathrm{coh}}\right\rangle^{2}\right]$, are useful when comparing with models and calculations, the functions $F_{\mathrm{K}}(Q), G_{\mathrm{K}}(r)$ etc., which are not scaled by $\left\langle b_{\mathrm{coh}}\right\rangle^{-2}$, permit a much more direct and unambiguous assessment of absolute data normalization when correcting experimental data. $F^{\mathrm{PDF}}(Q)$ and $G^{\mathrm{PDF}}(r)$ are more challenging in this regard as their respective $Q$ - and $r$-dependent asymptotes to the origin make determination of the low- $Q$ and low- $r$ trends less obvious 'by eye'. $S(Q)$ should also be used cautiously; although it is unitless this hides the fact that scattering factors are incorporated within the function, and even though many $S(Q)$ have a low- $Q$ limit that is close to zero this does not mean that zero is the limiting value by definition.

As a worked example of this, we show in Fig. 2 data from $\mathrm{BaTiO}_{3}$ measured on GEM (Hannon, 2005) at $15 \mathrm{~K}$ (Senn et al., 2016), which have been corrected using the Gudrun
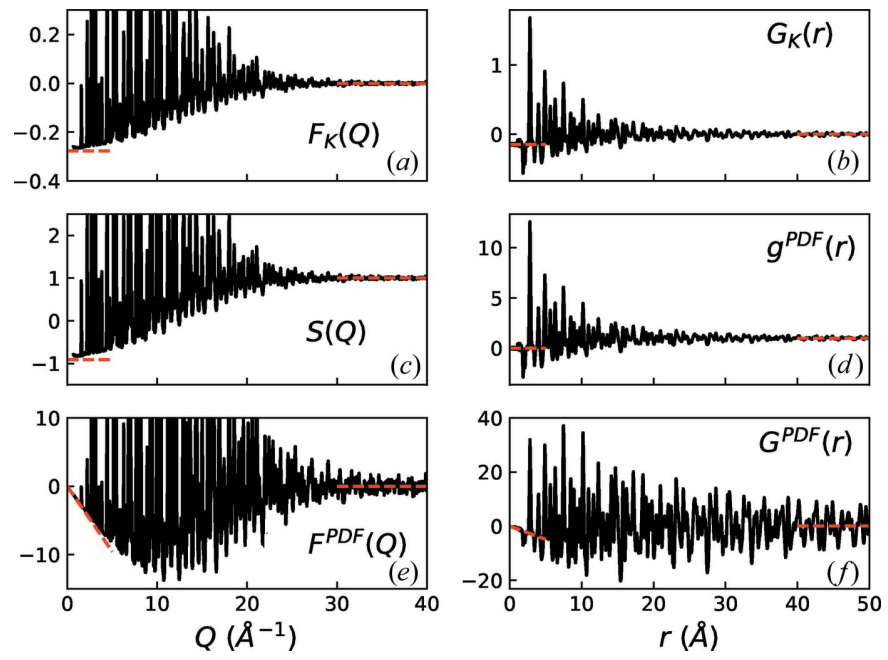

Figure 2

Experimental neutron total scattering functions from $\mathrm{BaTiO}_{3}$ at $15 \mathrm{~K}$ (Senn et al., 2016), using $\rho_{0}=0.0779$ atoms $\AA^{-3}$, determined from Rietveld refinement of the data. The various functions are defined using the equations given in Table 1 . The asymptotes at high and low $Q$ (or $r$, as appropriate) are highlighted using red dashed lines with values for reciprocal-space functions from Table 4; all real-space function limits are clearly seen to be 0 or 1 except for the low- $r$ limit of $G_{\mathrm{K}}(r)$, which is $-\left\langle b_{\text {coh }}\right\rangle^{2}=-0.145$ barn.
Table 4

List of reciprocal-space limits for selected materials with the assumption that $\eta=0$.

Although many materials have an $S(Q)$ with a limiting value at low $Q$ close to zero (e.g. $\mathrm{SiO}_{2}$ ), for materials containing elements with negative neutron coherent scattering lengths (e.g. $\mathrm{MnO}$ and $\left.\mathrm{BaTiO}_{3}\right) \lim _{Q \rightarrow 0} S(Q)$ is often far from zero.

\begin{tabular}{llcl}
\hline Material & Function & Low $Q$ & High $Q$ \\
\hline $\mathrm{MnO}$ & $S(Q)$ & -21.1 & 1 \\
& $F^{\mathrm{PDF}}(Q)$ & 0 & 0 \\
& $F_{\mathrm{K}}(Q)$ & -0.237 & 0 \\
$\mathrm{BaTiO}_{3}$ & $S(Q)$ & -0.911 & 1 \\
& $F^{\mathrm{PDF}}(Q)$ & 0 & 0 \\
& $F_{\mathrm{K}}(Q)$ & -0.277 & 0 \\
$\mathrm{SiO}_{2}$ & $S(Q)$ & -0.022 & 1 \\
& $F^{\mathrm{PDF}}(Q)$ & 0 & 0 \\
& $F_{\mathrm{K}}(Q)$ & -0.282 & 0 \\
\hline
\end{tabular}

program (Hannon et al., 1990; Soper, 2017). The Ti atoms in $\mathrm{BaTiO}_{3}$ have a negative neutron scattering length and the relevant average scattering constants are listed in Table 3 . The high- and low- $Q$ levels of the corrected differential scattering cross section (they should equal $\left\langle b_{\text {tot }}^{2}\right\rangle$ and tend to $\sim\left\langle b_{\text {tot }}^{2}\right\rangle-\left\langle b_{\text {coh }}^{2}\right\rangle=0.048$, respectively) are immediate indicators of the quality of the data correction [as are the limits of $F_{\mathrm{K}}(Q)$, i.e. after subtraction of $\left\langle b_{\mathrm{tot}}^{2}\right\rangle$; see Fig. 2(a)]. Typically when correcting data, a 'good' low- $Q$ limit is often much harder to achieve than a 'good' high- $Q$ limit. This is especially the case for time-of-flight neutron diffractometers where data corrections are usually more challenging in the lower- $Q$ regime. The low- $Q$ limit of $S(Q)$ should approximately equal $-\left\langle b_{\text {coh }}^{2}\right\rangle /\left\langle b_{\text {coh }}\right\rangle^{2}+1=-0.911$ [Fig. 2(c)]. The low- $r$ levels of $G_{\mathrm{K}}(r)=-\left\langle b_{\mathrm{coh}}\right\rangle^{2}$ and $g^{\mathrm{PDF}}(r)=0$ [Figs. $2(b)$ and $2(d)$, respectively]. Here a back-transform correction has been applied to direct these PDFs to their theoretical values for $r<$ $1 \AA$; encouragingly these values are maintained to much higher $r$, including in the gaps between the first few low- $r$ peaks. For completeness, plots of $F^{\mathrm{PDF}}(Q)$ and $G^{\mathrm{PDF}}(r)$ are shown in Figs. 2(e) and 2(f), respectively.

Finally, we note an inconsistency in the discussion of symmetric PDF peaks by Peterson et al. (2021). The different $r$ dependencies mean that it is not possible for all definitions of the PDF function to show symmetric peaks centred at the average pairwise distances. A symmetric and centred peak in $G^{\mathrm{PDF}}(r)$ will not be symmetric and centred in $g^{\mathrm{PDF}}(r)$ [equivalent to $G_{\mathrm{K}}^{\prime}(r)$ of Keen (2001)]. Symmetric peak fitting should only be carried out using PDF functions such as $G^{\mathrm{PDF}}(r)$ or $D(r)$ (Olds et al., 2018).

We have worked together on this corrigendum to try to ensure that these corrections to the Peterson et al. (2021) paper are clear and that the explicitly labelled functions herein mean that the relational expressions first established by Keen (2001) are not confused by the subtly redefined functions presented by Peterson et al. (2021), thus undermining the purpose of both papers. Total scattering notation has evolved over time since the equations were first conceived of by Zernike and Prins in 1927 (see a recent review and references 
therein; Keen, 2020), but it has stabilized over the past 20 years within the now mature total scattering community, in part through the cross-referencing of equations following Keen (2001). We as a community have a responsibility to ensure that we do not further compound any perceived notational confusion we might be trying to mitigate. Hopefully Keen (2001) and Peterson et al. (2021) with this corrigendum article will continue to provide the necessary clarity in total scattering function definitions.

\section{Acknowledgements}

The authors of this contribution acknowledge Daniel Olds, Marshall McDonnell and Katherine Page for feedback and comments during the production of this corrigendum. They are in complete agreement with the information provided herein.

\section{Funding information}

Funding for this research was provided by the US Department of Energy, Office of Science (contract No. DE-AC0500OR22725).

\section{References}

Bhatia, A. \& Thornton, D. (1970). Phys. Rev. B, 2, 3004-3012.

Bowron, D., Finney, J., Hallbrucker, A., Kohl, I., Loerting, T., Mayer, E. \& Soper, A. (2006). J. Chem. Phys. 125, 194502.

Cusack, N. (1987). The Physics of Structurally Disordered Matter: an Introduction. Bristol: Adam Hilger/IOP Publishing.

Fischer, H. E., Barnes, A. C. \& Salmon, P. S. (2006). Rep. Prog. Phys. 69, 233-299.

Hannon, A. (2005). Nucl. Instrum. Methods Phys. Res. A, 551, 88-107.

Hannon, A., Howells, W. \& Soper, A. (1990). Inst. Phys. Conf. Ser. 107, 193-211.

Keen, D. A. (2001). J. Appl. Cryst. 34, 172-177.

Keen, D. A. (2020). Crystallogr. Rev. 26, 143-201.

McGreevy, R. \& Mitchell, E. (1982). J. Phys. C. Solid State Phys. 15, 5537-5550.

Olds, D., Saunders, C. N., Peters, M., Proffen, T., Neuefeind, J. \& Page, K. (2018). Acta Cryst. A74, 293-307.

Peterson, P. F., Olds, D., McDonnell, M. T. \& Page, K. (2021). J. Appl. Cryst. 54, 317-332.

Sears, V. F. (1992). Neutron News, 3(3), 26-37.

Senn, M., Keen, D., Lucas, T., Hriljac, J. \& Goodwin, A. (2016). Phys. Rev. Lett. 116, 207602.

Soper, A. (2017). Gudrun - Routines for Reducing Total Scattering Data, https://www.isis.stfc.ac.uk/Pages/Gudrun.aspx. 